\title{
Problèmes liés à la structure génétique des populations naturelles
}

\author{
par E. BOESIGER \\ Centre National de Recherche Scientifique, F. 91 -Gif-sur-Yvette
}

\begin{abstract}
Résumé
L'application des méthodes de lutte génétique présente encore de nombreuses difficultés. Des recherches approfondies de la structure génétique des populations sont nécessaires pour éviter dans l'avenir les échecs. L'étude des populations naturelles de Drosophilidae prouve l'existence d'un nombre pratiquement illimité de variantes génétiques, portant sur la morphologie exiérieure, les caractères biométriques, les gènes létaux, les types structuraux des chromosomes, les mutants biochimiques et des caractères de comportement.
\end{abstract}

Les populations répondent à la complexité d'un milieu donné et à la différenciation géographique de différents biotopes par la multiplicité des génotypes de chaque population et par leur différenciation écologique dans différentes régions.

Les conditions génétiques déterminant la vigueur des organismes, essentielle pour le succès de la lutte génétique, sont discutées.

Quelques exemples meıtent en évidence les phénomènes de l'homéostase du développement et de l'homéostase génétique dont dépendent la capacité d'adaptation des organismes et des populations aux conditions multiples et variables des milieux.

L'attention est attirée tout particulièrement sur l'importance primordiale du comportement sexuel et d'autres facteurs éthologiques pour la lutte génétique. 


\section{Summary}

The application of the methods of genetical control presents still numerous difficulties. Precise investigations about the genetical structure of the populations are needed for to avoid failures in the future. The study of natural populations of Drosophilidae shows the existence of a practically illimited number of genetical variants, concerning the external morphology, the biometrical traits, lethal genes, the structural types of the chromosomes, biochemical mutants and behavioral traits.

The populations are responding to the complexity of a given environment and to the geographical differentiation of different places by the multiplicity of the genotypes of each population and by their ecological differentiation in different regions.

The genetical conditions responsable for the vigor of the organisms, essential for the success of the genetical control, are discussed.

Some examples are given for the developmental and the genetical homeostasis phenomena which are determinating the adaptability of organisms and populations to the multiple and varying conditions of the environments.

A special attention is given to the high importance of the sexual behaviour and other ethological characters for the genetical control.

\section{Introduction}

La lutte contre les Arthropodes en général et contre les Insectes hématophages en particulier a jusqu'à présent presque exclusivement été menée à l'aide d'insécticides. L'utilisation massive de ces substances toxiques a au moins trois inconvénients. Elle provoque une pollution dangereuse des eaux et des terres, elle coûte cher et risque d'être inefficace à long terme, parce que les populations d'Insectes se reconstituent à partir d'individus génétiquement résistants.

Les méthodes de lutte biologique ont le grand avantage de ne pas être dangereuses, ni pour l'homme ni pour d'autres espèces. Elles coûtent moins cher, et on peut espérer qu'elles soient efficaces à long terme.

Le principe de la lutte génétique est simple, mais l'application présente de nombreuses difficultés. Elles proviennent de la complexité de la constitution génétique des populations naturelles, de leur différenciation écologique et éthologique et de l'action puissante de la sélection naturelle, qu'il est difficile d'imiter dans les élevages de laboratoire. 
Les principales méthodes de lutte génétique sont:

$1^{\circ}$ L'introduction massive de mâles stériles dans les populations naturelles, pour obtenir un taux réduit de femelles pondant des œufs fécondés.

$2^{\circ}$ L'introduction massive de mâles d'une espèce proche, pour obtenir des hybridations interspécifiques, qui produisent des descendan:s stériles, ou qui empêchent la production de descendants.

$3^{\circ} \mathrm{La}$ stérilisation des femelles et des mâles de la population naturelle par des chimiostérilisants.

$4^{\circ}$ L'introduction massive de mâles porteurs de facteurs létaux, associés à des locus de distorsion de ségrégation, ou porteurs d'aberrations chromosomiques, notamment de transiocations, pour réduire la vitalité de la population naturelle.

$5^{\circ}$ L'introduction massive de mâles porteurs de gènes délétères pour réduire la vitalité de la population naturelle.

Cette technique, proposée par Knipling (1960), permettrait l'introduction de gènes provoquant l'inaptitude à la diapause, l'inaptitude au vol, l'absence de la sécrétion qui colle les œufs au support ou la modification des pièces buccales. Il sera relativement facile de trouver d'autres mutations délétères.

$6^{\circ}$ L'augmentation du taux de masculinité.

$7^{\circ}$ L'utilisation de l'incompatibilité cytoplasmique.

Nous sommes bien obligés d'admettre que l'application opérationnelle de la lutte génétique a parfois donné lieu à des résultats décevants. Ces échecs ne doivent pas nous décourager. La lutte biologique est la seule voie acceptable et efficace à long terme. Il faut tout faire pour la réussir. Les difficultés sont du domaine de la génétique des populations et de la génétique écologique. Les méthodes de lutte génétique sont fondées sur l'introduction massive dans les populations naturelles d'individus élevés au laboratoire et portant des caractères génétiques particuliers. Il est donc indispensable de discuter des phénomènes et des mécanismes de la génétique des populations, sous leur aspect écologique, intervenant quand des animaux sont introduits dans une population naturelle.

Les travaux, qui nous font connaître ces mécanismes, ont plus souvent été effectués avec des Drosophiles, des Lépidoptères, des Crustacés et des Cailles qu'avec des Moustiques. Cela n'a aucun inconvénient, puisque les mécanismes que nous évoquerons existent certainement aussi chez les Moustiques et chez d'autres Arthropodes hématophages. Avant l'application pratique une adaptation spécifique sera pourtant nécessaire dans chэque ras.

Pour réussir la lutte génétique, il ne suffit pas de choisir une bonne méthode, de produire les animaux au laboratoire et de les relâcher dans la nature. Il est indispensable que les individus élevés au laboratoire survivent ensuite dans la population naturelle et y accomplissent la tâche qui leur est assignée.

Pour éviter les échecs, il faut connaître la constitution génétique des populations naturelles à éliminer, tenir compte de la différenciation géographique de ces popula- 
tions, conférer aux individus qu'on introduit dans les populations naturelles une vigueur au moins égale à celle des individus de la population naturelle, choisir un génotype qui leur assure une gamme suffisamment large de capacités d'adaptation au milieu naturel, et s'assurer que les individus à relâcher dans la nature ont un comportement sexuel qui leur permettra d'introduire leurs gènes dans la population naturelle ou d'assurer la stérilité des femelles.

\section{Structuration génétique et hétérogénéité des populations naturelles}

Les populations naturelles d'une espèce ne sont pas uniformes. Le "phénotype sauvage », le «type » de l'espèce, marqué dans les collections d'une pastille rouge, a une certaine utilité taxonomique, mais il n'a pas le moindre intérêt en génétique des populations, et il est une fiction dangereuse dans la lutte génétique. Il est indispensable de savoir que les populations naturelles sont totalement hétérogènes, à un point tel qu'il n'existe pas deux individus avec un génotype identique. Des considérations théoriques, fondées sur de nombreuses observations, nous permettent de prendre une position aussi extrême.

Lewontin et Hubby (1966) font une estimation du degré d'hétérozygotie des populations naturelles de Drosophila pseudoobscura à partir d'un relevé des locus hétérozygotes pour des gènes déterminant certaines enzymes. Ils comptent avec $15 \%$ de locus hétérozygotes, ce qui est probablement une sous-estimation. On a souvent avancé le chiffre de 10.000 locus pour une Drosophile, chiffre qui semble sous-estimé. Si nous supposons pour chaque locus polymorphe deux allèles seulement, cela donne trois génotypes par locus: AA, Aa et aa. Le nombre de génotypes, théoriquement possibles à partir de ces données, est énorme : $3^{1500}$. Ce chiffre dépasse de très loin le nombre de toutes les Drosophiles, qui ont existé et qui existeront dans l'avenir.

Certes, ces combinaisons géniques, théoriquement possibles, ne se produisent pas toutes, et celles qui le sont à l'état de zygotes, ne sont pas toutes viables. En plus, les différences génotypiques ne s'expriment pas toutes par des différences phénotypiques.

Les travaux sur la constitution génétique des populations naturelles de Drosophilides prouvent l'existence d'un nombre pratiquement illimité de variantes génétiques (Boesiger, $1966 a$ ). Il s'agit de systèmes génétiques déterminant des caractères biométriques, des caractères morphologiques extérieurs, des caractères biochimiques, des gènes létaux, des caractères anatomiques, des types structuraux de chromosomes et des caractères de comportement.

Parmi de nombreux autres faits, on peut mentionner les fréquences de Mouches de type mutant dans les populations naturelles de D. melanogaster et de D. subobscura, présentées dans le tableau $\mathrm{n}^{\circ} 1$ (Boesiger, 1962 ; Pentzos, Boesiger et Kanellis, 1967 ; Dubinin et coll., 1937). 
Tableau 1. - Fréquence des individus de type mutant dans des populations naturelles de $D$. melanogaster et de $D$. subobscura

\begin{tabular}{|c|c|c|c|c|c|}
\hline \multirow{2}{*}{ Espèce } & \multirow{2}{*}{ Population } & \multirow{2}{*}{$\begin{array}{l}\mathrm{N} \text { de mouches } \\
\text { observées }\end{array}$} & \multicolumn{3}{|c|}{$\begin{array}{l}\text { Pourcentage des mouches } \\
\text { de type mutant }\end{array}$} \\
\hline & & & 우 & $\hat{\jmath}$ & $\overline{\mathrm{m}}$ \\
\hline \multirow[t]{4}{*}{ D. melanogaster. } & Banyuls A & 1.207 & 7,21 & 3,60 & 4,80 \\
\hline & Banyuls B & 772 & 10,42 & 3,44 & 6,48 \\
\hline & Banyuls U & 3.990 & 9,48 & 4,44 & 6,44 \\
\hline & Gelendzhik & 10.000 & 一 & - & 6,84 \\
\hline D. subobscura . & Thasos & 1.187 & 9,50 & 5,90 & 7,41 \\
\hline
\end{tabular}

En élevant la descendance de femelles de Drosophiles capturées dans une population naturelle et en croisant des frères et sœurs de la $F_{1}$, on peut déterminer en $F_{2}$ la fréquence des gènes mutants qui se trouvaient à l'état hétérozygote chez ces femelles. Le tableau $\mathrm{n}^{\circ} 2$ présente quelques-unes de ces observations (Boesiger, 1962; Pentzos, Boesiger et Kanellis, 1967).

Tableau 2. - Fréquence de gènes mutants se trouvant à l'état hétérozygote chez des femelles de Drosophilides dans les populations naturelles

\begin{tabular}{|c|c|c|c|}
\hline Espèce & Population & $\begin{array}{l}\mathrm{N} \text { de femelles } \\
\text { analysées }\end{array}$ & $\begin{array}{l}\overrightarrow{\mathrm{m}} \text { du } \mathrm{N} \text { de mutants } \\
\text { à l'état hétérozy- } \\
\text { gote par femelle }\end{array}$ \\
\hline D. melanogaster ... & $\begin{array}{l}\text { Bâle } 1952 \\
\text { Banyuls } 1952 \\
\text { Banyuls } 1953 \\
\text { Domme } 1957 \\
\text { Nimba, Pavia } 1956\end{array}$ & $\begin{array}{r}122 \\
160 \\
218 \\
95 \\
61\end{array}$ & $\begin{array}{l}2,90 \\
2,54 \\
4,45 \\
4,36 \\
3,52\end{array}$ \\
\hline D. subobscura $\ldots$. & $\begin{array}{l}\text { Litochoron } 1966 \\
\text { Samothraki } 1966 \\
\text { Thasos } 1966\end{array}$ & $\begin{array}{r}47 \\
48 \\
189\end{array}$ & $\begin{array}{l}4,60 \\
4,40 \\
3,50\end{array}$ \\
\hline
\end{tabular}

On trouve également de nombreux gènes létaux dans les populations naturelles de Drosophilides. Ives (1945) relève dans une population de D. melanogaster de Floride, dans $44,1 \%$ de Mouches au moins, un gène létal sur le deuxième chromosome. Chez D. pseudoobscura Dobzhansky et Spassky (1953) comptent $33 \%$ de chromosomes II, $25 \%$ de chromosomes III et $25,9 \%$ de chromosomes IV qui portent au moins un gène létal ou semi-létal. 
Bien que nous ne disposions pas chez d'autres organismes des souches adéquates, permettant des recensements précis, l'élevage en stricte consanguinité d'individus prélevés dans des populations naturelles, prouve l'existence d'un lourd fardeau génétique.

L'hétérogénéité des populations naturelles de Drosophiles n'est pas chaotique. Les importants travaux de Dobzhansky (voir Dobzhansky et Epling, 1944 ; Dobzhansky et Boesiger, 1968) sur les fréquences de différents types structuraux des chromosomes de D. pseudoobscura, D. persimilis, D. willistoni et $D$. paulistorum permettent d'affirmer qu'il existe une adaptation écologique entre le pool des génotypes d'une population et les conditions de son milieu.

Les patrons des fréquences de différents types structuraux sont caractéristiques pour chaque région. On constate des fluctuations réversibles au cours des saisons, qui représentent sans doute une adaptation saisonnière. Il existe aussi une évolution irréversible de ces fréquences à long terme, qui correspond peut-être à des remaniements du contenu génique de certaines inversions.

Chaque biotope est complexe. Il présente différentes conditions de température, d'humidité, de lumière; différents aliments, différents parasites, différents prédateurs et différents types de compétition avec d'autres organismes. Aux conditions multiples et variables d'un milieu correspond nécessairement la complexité du pool des génotypes qu'on trouve dans une localité. Le polymorphisme génétique est une des expressions de cette hétérogénéité. La différenciation des espèces en races géographiques, dans une large mesure déterminée par des conditions écologiques particulières, est une autre expression de l'hétérogénéité des espèces.

Est-il besoin d'insister sur l'importance de la connaissance de la structure génétique de chaque population naturelle dans laquelle on essaie d'introduire des individus élevés au laboratoire. Les animaux qu'on lâche dans une population naturelle doivent être écologiquement adaptés aux conditions particulières de chaque population pour survivre. Il ne suffit malheureusement pas de trouver un génotype optimal et unique, reproduit au laboratoire à des centaines de millions d'exemplaires. Il sera nécessaire de relâcher dans chaque biotope une population hétérogène, représentant un éventail de génotypes adéquatement ajusté aux conditions multiples et variables de l'habitat particulier. Il sera nécessaire de travailler avec des pools de génotypes différents pour chaque région à traiter.

\section{Conditions génétiques déterminant la vigueur des organismes}

Une autre condition, très importante pour le succès de la lutte génétique, est la vigueur adéquate des individus qu'on cherche à introduire dans une population naturelle. Dans ce domaine il est inévitable de mentionner les deux écoles qui s'opposent. En simplifiant le problème, on pourrait dire que l'une des deux tendances, la plus ancienne, préconise la création de populations homogènes. La sálection naturelle aurait tendance à éliminer à chaque locus les allèles de valeur sélective inférieure en faveur 
du meilleur allèle disponible. Dans le cas idéal, jamais atteint à cause de la production continuelle de mutations, presque toutes délétères, tous les individus d'une population seraient homozygotes à tous les locus pour le meilleur allèle disponible. Le but de la sélection artificielle devrait être l'élimination de tous les gènes délétères. Quand il s'agit de produire de grands nombres d'animaux, par exemple pour la lutte génétique, ces lots expérimentaux devraient être homogènes, reproduisant le génotype homozygote idéal, exempt de tout gène délétère.

L'autre tendance s'est développée sous l'influence de l'étude approfondie de la structure génétique des populations naturelles, ainsi que de l'analyse expérimentale des phénomènes de l'hétérosis et de l'homéostase génétique. Il ne s'agit pas de présenter dans ce rapport en détail les faits et les hypothèses, qui permettraient de confronter les deux tendances. On trouvera des éléments pour cette discussion dans des exposés assez récents (Dobzhansky, 1951 ; Lerner, 1954 ; Boesiger, 1962, 1965, $1969 a, 1969 b, 1969 c$; Ford, 1964, 1965 ; Dobzhansky et Boesiger, 1968).

Le succès de la lutte génétique dépendra dans une large mesure des méthodes de génétique des populations, qui seront fondamentalement différentes selon le choix de l'une ou de l'autre des deux tendances principales. Pour cette raison c'est un devoir du rapporteur de prendre position, et d'attirer l'attention sur quelques points essentiels de la tendance en génétique des populations que nous préconisons pour la lutte génétique.

Les populations naturelles sont très hétérogènes, composées d'individus qui ont un degré élevé d'hétérozygotie. Cette hétérogénéité et ces degrés élevés d'hétérozygotie ne sont pas des défauts, des imperfections de la nature, des preuves de l'inefficacité de la sélection naturelle. Bien au contraire: la sélection naturelle favorise les individus hétérozygotes à cause de leur vigueur hybride et elle maintient l'hétérogénéité des populations parce qu'elle assure une homéostase génétique indispensable pour des raisons écologiques.

Les organismes hétérozygotes manifestent une plus grande vigueur, une fécondité et une fertilité plus élevées ainsi qu'une meilleure résistance contre les parasites et contre des conditions défavorables du milieu.

Seules les populations hétérogènes sont capables de vivre dans des conditions de milieu hétérogènes à un moment donné et variables dans le temps. L'hétérogénéité offre le potentiel génétique nécessaire à la population pour rester adaptée malgré les fluctuations du milieu et elle assure la production d'individus hétérozygotes.

Un gène n'a nullement une valeur sélective constante, positive ou négative. Un gène hautement délétère à l'état homozygote, par exemple un gène létal, peut avoir des avantages importants à l'état hétérozygote et dans certaines conditions. La valeur sélective d'un gène changera selon l'ensemble des autres gènes de l'organisme, sélon la fréquence du génotype particulier dans la population et selon les conditions écologiques. Puisque les conditions du milieu, la composition de la population, la composition de l'ensemble de la flore et de la faune, qui font partie du milieu pour une population donnée, ne sont jamais uniformes, ni stables, ce sera dans tous les cas un 
avantage pour l'ensemble d'une population de disposer d'un grand éventail génétique, offrant de larges possibilités de riposte, bien que cela implique la présence de nombreux gènes délétères.

Certains échecs de la lutte génétique s'expliquent par le manque de vigueur des animaux produits au laboratoire. Les facteurs principaux qui déterminent une bonne compétitivité et une large capacité d'adaptation, sont très similaires chez différents organismes. Puisque nous disposons d'études expérimentales sur le déterminisme génétique de la vigueur chez les Drosophiles et chez les Cailles japonaises, il convient de résumer les principaux résultats obtenus chez ces organismes.

\section{$1^{\circ}$ Vigueur sexuelle des mâles de Drosophiles.}

On peut évaluer la vigueur sexuelle des mâles de Drosophiles de plusieurs manières. Quand on place dans une cage à population le même nombre de deux types de mâles avec un seul type de femelles vierges, pendant 48 beures, l'étude de la descendance de chacune des femelles permet de savoir, si l'un des deux types de mâles féconde en moyenne plus de femelles que l'autre. Le tableau $\mathrm{n}^{\circ} 3$ présente une série d'expériences de ce type.

Tableau 3. - Comparaison de la vigueur sexuelle des mâles « vermillon » et « cinnabar» de D. melanogaster (Boesiger, 1962)

\begin{tabular}{|c|c|c|c|c|}
\hline \multirow{2}{*}{$\begin{array}{c}\text { Nombre total } \\
\text { de }+\%\end{array}$} & \multicolumn{3}{|c|}{ 우 Fécondées par des $\hat{o} \hat{o}$} & \multirow{2}{*}{$\begin{array}{c}\% \text { de } 9 \text { \% } \\
\text { fécondées par } \\
\text { les } \hat{\delta} \hat{\delta} \text { \&n }\end{array}$} \\
\hline & «n & 《V & 《cn》 et $\ll v »$ & \\
\hline \multicolumn{5}{|c|}{ Femelles de type «vermillon» } \\
\hline $\begin{array}{r}74 \\
163 \\
168 \\
176 \\
253 \\
230\end{array}$ & $\begin{array}{r}66 \\
140 \\
139 \\
144 \\
214 \\
167\end{array}$ & $\begin{array}{r}2 \\
3 \\
11 \\
21 \\
7 \\
15\end{array}$ & $\begin{array}{l}2 \\
6 \\
2 \\
0 \\
6 \\
1\end{array}$ & $\begin{array}{l}95,71 \\
95,97 \\
92,10 \\
87,27 \\
95,59 \\
91,53\end{array}$ \\
\hline 1.064 & 1.870 & 59 & 17 & $92,86 \pm 1,40$ \\
\hline \multicolumn{5}{|c|}{ Femelles de type «cinnabar» } \\
\hline $\begin{array}{l}186 \\
173 \\
277 \\
139 \\
100 \\
246 \\
223\end{array}$ & $\begin{array}{r}153 \\
94 \\
113 \\
66 \\
77 \\
172 \\
139\end{array}$ & $\begin{array}{r}13 \\
2 \\
10 \\
5 \\
6 \\
15 \\
13 \\
13\end{array}$ & $\begin{array}{r}11 \\
1 \\
0 \\
2 \\
8 \\
8 \\
5\end{array}$ & $\begin{array}{l}89,54 \\
97,42 \\
91,86 \\
91,78 \\
89,01 \\
90,25 \\
90,12\end{array}$ \\
\hline 1.344 & 814 & 64 & 35 & $91,07 \pm 1,08$ \\
\hline
\end{tabular}


Puisque le nombre des deux types de mâles était identique dans chacune des populations expérimentales, la moitié des femelles aurait dû être fécondée par l'un des deux types de mâles, et l'autre moitié, par l'autre type de mâles, si leur activité sexuelle était la même. Or, on trouve un écart considérable par rapport à la panmixie. La grande majorité des femelles, qu'elles soient de type vermillon ou de type cinnabar, est fécondée par les mâles cinnabar. Une longue analyse expérimentale que nous ne présenterons pas ici (Boesiger, 1962), prouve que ce n'est pas le gène cinnabar lui-même qui confère aux mâles de cette souche une valeur sélective élevée dans la compétition sexuelle, mais le haut degré d'hétérozygotie de cette souche, marquée par le gène cinnabar. Cette souche était en effet fraîchement dérivée de Mouches capturées dans une population naturelle et maintenue au laboratoire en évitant l'augmentation de la consanguinité, alors que la souche marquée par le gène vermillon était élevée depuis de très longues années en forte consanguinité au laboratoire.

L'observation directe du comportement sexuel des deux types de mâles révèle également de grandes différences, qui expliquent la plus grande compétitivité des mâles de la souche cinnabar.

Le temps d'attente jusqu'au début de la parade nuptiale, à partir de la mise en contact des couples dans une chambre d'observation, est en moyenne plus court pour les mâles cinnabar.

Le temps d'attente jusqu'au début de la copulation, observé dans les mêmes conditions, est en moyenne beaucoup plus court pour les mâles cinnabar. En présence de femelles de type vermillon, $48,3 \%$ des mâles de type cinnabar copulent pendant les cinq premières minutes de l'observation alors que $12,9 \%$ seulement des mâles vermillon ont copulé dans le même temps.

On constate d'autres différences entre les deux types de mâles. Les femelles refusent presque toujours, au début de la parade nuptiale, le contact avec le mâle. Quand un mâle s'approche d'une femelle, celle-ci repousse le mâle, parfois en donnant de vigoureux coups de pattes. Habituellement elle s'éloigne ensuite du mâle. Dans ces conditions, les mâles hétérozygotes de la souche cinnabar recherchent la femelle très activement et recommencent une nouvelle parade nuptiale intense et persistante. Les mâles de la souche vermillon, en revanche, perdent le contact avec la femelle. Ils effectuent une parade nuptiale peu intense et souvent interrompue.

Après une certaine durée de la parade nuptiale, les mâles essaient brusquement de monter la femelle et de procéder à la copulation. Quand la stimulation sexuelle de la femelle n'a pas atteint à ce moment le niveau nécessaire, le mâle est repoussé. De ce fait, on constate un certain nombre d'essais infructueux de copulation. Le recensement de ces essais de copulation permet une évaluation de l'agressivité sexuelle des mâles. Le tableau $\mathrm{n}^{\circ} 4$ donne des résultats d'une série d'observations concernant ces essais de copulation. Il montre que les mâles de la souche cinnabar ont une agressivité sexuelle environ quatre fois plus grande que celle des mâles de la souche consanguine vermillon.

On constate aussi une différence notable du comportement des femelles des deux souches. Les femelles de la souche hétérozygote résistent plus longtemps que les 
femelles de la souche consanguine et provoquent ainsi un plus grand nombre d'essais de copulation. $\mathrm{n}$ convient de tenir compte de ces faits dans la production d'animaux destinés à la lutte génétique.

Un taux satisfaisant de fécondation obtenu avec des femelles d'une souche relativement consanguine élevée au laboratoire, ne prouve pas que les mâles ainsi évalués produiraient les mêmes résultats avec des femelles beaucoup plus résistantes d'une population naturelle.

Tableau 4. - Comparaison du nombre des essais de copulation des mâles cinnabar et vermillon (Boesiger, 1962)

\begin{tabular}{|c|c|c|c|c|}
\hline \multirow{2}{*}{$\begin{array}{l}\text { Nombre } \\
\text { d'essais }\end{array}$} & \multicolumn{2}{|c|}{ Femelles vermillon } & \multicolumn{2}{|c|}{ Femelles cinnabar } \\
\hline & $\mathrm{n} \delta \mathrm{v}$ & $\mathrm{n} \partial \hat{\mathrm{cn}}$ & $\mathrm{n} \hat{\delta} \mathrm{v}$ & $\mathrm{n} \delta \mathrm{cn}$ \\
\hline 0 & 14 & 2 & 7 & 6 \\
\hline 1 & 10 & 11 & 9 & 3 \\
\hline 2 & 4 & 1 & 0 & 4 \\
\hline 3 & 3 & 4 & 1 & 1 \\
\hline 4 & 0 & 4 & 1 & 2 \\
\hline 5- 8 & 0 & 3 & 0 & 5 \\
\hline $9-12$ & 0 & 3 & 0 & 1 \\
\hline 17 & 0 & 0 & 1 & 0 \\
\hline 21 & 0 & 1 & 0 & 0 \\
\hline 35 & 0 & 0 & 0 & 1 \\
\hline $\begin{array}{l}\text { Total ....... } \\
\text { Moyenne } \ldots\end{array}$ & $\begin{array}{l}27 \\
0,88\end{array}$ & $\begin{array}{c}114 \\
3,93\end{array}$ & $\begin{array}{l}33 \\
1,35\end{array}$ & $\begin{array}{c}101 \\
4,39\end{array}$ \\
\hline
\end{tabular}

Quand on compte dans de petites populations expérimentales le nombre de femelles fécondées au cours de 48 heures par différents types de mâles, on trouve des différences de fréquences considérables entre souches (Boesiger, 1962). Les mâles d'une souche mutante «vestigial» fécondent dans ces conditions $9,52 \%$ des femelles, ceux d'une souche fraîchement dérivée d'une population naturelle de Bourgogne fécondent 93,33\% des femelles. En moyenne, les mâles de souches élevées depuis longtemps au laboratoire fécondent $43,02 \%$ des femelles, alors que les mâles de souches dérivées de Mouches capturées dans des populations naturelles fécondent $73,05 \%$ des femelles.

Une preuve plus directe de l'influence primordiale de l'hétérozygotie sur la vigueur des mâles est fournie par la comparaison du taux de femelles fécondées par des mâles de deux souches très consanguines de Drosophiles avec celui de mâles hybrides obtenus par le croisement des deux souches.

Dans les conditions expérimentales déjà décrites ci-dessus, les mâles d'une souche mutante «forked»fécondent $23,78 \%$ des femelles, ceux d'une souche mutante «se- 
pia » fécondent $28,31 \%$ des femelles. Les mâles hybrides, issus du croisement des deux souches fécondent $79,59 \%$ des femelles. Les mâles hétérozygotes sont donc beaucoup plus compétitifs.

On trouve également un très grand décalage entre le nombre de descendants engendrés après une seule copulation par les mâles des souches consanguines «sepia » et « forked » déjà mentionnées et les mâles hybrides (Boesiger, 1963). Or cette capaci.é élevée de reproduction, qui n'est pas recherchée elle-même dans la lutte génétique, dans la plupart des cas, est liée à la vigueur générale des mâles, donc à leur compétitivitś. Elle est un indicateur de la vigueur générale des individus, indispensable pour la lutte génétique.

\section{$2^{\circ}$ Comparaison de Drosophiles d'élevage avec celles des populations naturelles.}

La comparaison de la survie de femelles de Drosophila melanogaster, capturées dans des populations naturelles, avec celle de leur descendance, a permis de mettre en évidence des différences notables (Boesiger, 1968 et travaux en cours). La moyenne de la durée de survie est de 54,5 jours pour un lot de femelles capturées à Banyuls, de 66,4 jours pour un lot de femelles d'Argelès et de 47,3 jours seulement pour les descendants du premier échantillon. Pour les deıx premiers groupes d'animaux prélevés dans la nature, la fraction de la durée de vie antérieure à la capture n'entre pas en ligne de compte pour la moyenne. La différence est donc plus importante que les chiffres ne le montrent.

Il est possible que les conditions d'élevage au laboratoire, et notamment l'alimentation larvaire, soient moins bonnes que celles que les larves rencontrent dans la nature, ce qui explique peut-être partiellement la supériorité des Mouches capturées dans les populations naturelles. A partir du moment de leur capture, les Mouches de la population naturelle ont évidemment été maintenues dans les mêmes conditions que leur descendance.

Il est pourtant plausible qu'une autre hypothèse explique au moins partiellement la supériorité des Mouches capturées dans la nature. Au laboratoire, nous réussissons à élever la majorité des larves, alors que la forte pression de la sélection naturelle élimine une fraction importante des larves et des imagos dans les populations naturelles. Les Mouches capturées dans la nature représentent donc un choix des meilleurs génotypes. Puisque les populations naturelles sont hétérogènes et les individus hétérozygotes, leurs descendances reconstituent à chaque génération un éventail de génotypes, présentant tous les degrés de valeurs sélectives.

Il est donc très probable qu'un lot d'animaux élevés au laboratoire présente une valeur sélective moyenne inférieure à celle qu'on trouve dans les populations naturelles. Cette différence doit être particulièrement importante chez les Insectes et chez d'autres organismes qui subissent dans la nature des taux élevés d'élimination par la sélection naturelle. Ce phénomène a une grande importance pour le choix des techniques de production d'organismes qui doivent servir à la lutte génétique. 


\section{Homéostase génétique et capacité d'adaptation}

L'étude de la constitution génétique de populations naturelles de Drosophilides, de Crustacés, de Lépidoptères, de Gastéropodes, etc. révèle leur grande hétérogénéité. Les populations naturelles sont composées de nombreux génotypes, parce que les conditions du milieu sont complexes et variables. Une population locale se trouve en présence de conditions physiques variables, de différents aliments, différents prédateurs et parasites. Or nous savons que les individus hétérozygotes peuvent affronter une gamme plus large de conditions physiques et que les populations hétérogènes sont plus aptes à maintenir les normes d'adaptation (Boesiger, 1969 b). Il est important pour le succès de la lutte génétique, que les organismes et les populations à relâcher dans la nature présentent un degré d'homéostase de développement et d'homéostase génétique suffisant pour survivre dans les conditions naturelles. C'est une des conditions qui assurent la compétitivité des organismes à relâcher et la régularité des résultats.

Nous ne disposons pas encore de connaissances sur l'homéostase génétique des Arthropodes vecteurs. Puisqu'il s'agit de phénomènes biologiques de valeur générale, leur mise en évidence chez d'autres organismes doit servir de modèle.

Chez Drosophila melanogaster le nombre de descendants engendrés après une seule copulation est beaucoup plus élevé pour des mâles hybrides, issus du croisement de deux souches à forte consanguinité. La variation du nombre de descendants des mâles est beaucoup plus faible chez les mâles hétérozygotes (Boesiger, 1963). Le tableau $n^{\circ} 5$ résume à titre d'exemple quelques résultats expérimentaux.

Tableau 5. - Variance du nombre de descendants engendrés après une seule copulation chez D. melanogaster

\begin{tabular}{|c|c|c|c|}
\hline Croisements & $\begin{array}{c}\text { Nombre } \\
\text { de } \\
\text { couples }\end{array}$ & $\begin{array}{c}\text { Nombre moyen } \\
\text { de descendants } \\
\text { par } \subsetneq\end{array}$ & $\begin{array}{c}\text { Coefficient } \\
\text { de } \\
\text { variation }\end{array}$ \\
\hline $\begin{array}{l}q \text { Aloxe } \times \sigma^{2} \text { sepia }(\text { souche consan- } \\
\text { guine }) \ldots \ldots \ldots \ldots \ldots \ldots \ldots \ldots \ldots \ldots \ldots\end{array}$ & 48 & $16.5 \pm 3.55$ & 148.9 \\
\hline $\begin{array}{l}q \text { Aloxe } \times \delta \text { forked (souche consan- } \\
\text { guine) } \ldots \ldots \ldots \ldots \ldots \ldots \ldots \ldots \ldots \ldots \ldots\end{array}$ & 42 & $61,5 \pm 14,56$ & 153,3 \\
\hline$q$ Aloxe $\times \delta$ sepia/forked (hybrides) & 45 & $399,3 \pm 18,09$ & 30,3 \\
\hline+ Aloxe $\times \sigma^{*}$ forked/sepia (hybrides) & 41 & $488,4 \pm 16,44$ & 21,5 \\
\hline o sepia $\times \hat{o}$ forked $/$ sepia $\ldots \ldots \ldots$ & 37 & $124,8 \pm 22,74$ & 110,8 \\
\hline$q$ forked $\times \hat{o}$ forked/sepia $\ldots \ldots$ & 39 & $159,4 \pm 16,32$ & 63,9 \\
\hline o forked/sepia $\times \delta$ forked/sepia $\ldots$ & 39 & $394,2 \pm 13,43$ & 21,2 \\
\hline \% sepia/forked $\times$ o sepia/forked ... & 39 & $405,6 \pm 15,12$ & 23,2 \\
\hline
\end{tabular}


Chez la Caille japonaise la variance du taux d'éclosion des œufs augmente en relation avec le degré de consanguinité, alors que la moyenne du taux d'éclosion baisse (Boesiger, 1969).

Le tableau 6 donne les résultats d'incubations effectuées dans des conditions normales.

Tableau 6. - Taux d'éclosion des œufs, après incubation normale, chez la Caille japonaise

\begin{tabular}{|c|c|c|c|c|}
\hline Génération & 《F & $\mathrm{N}$ & $\begin{array}{l}\text { Pour } 100 \\
\text { éclosion }\end{array}$ & $\begin{array}{l}\text { Coefficient } \\
\text { de variation }\end{array}$ \\
\hline$P \ldots \ldots \ldots$ & 0 & 269 & 81,3 & 10,76 \\
\hline $\mathrm{F}_{1} \ldots \ldots \ldots$ & 0,25 & 267 & 70,7 & 24,78 \\
\hline $\mathrm{F}_{2} \ldots$ & 0,37 & 278 & 43,6 & 25,38 \\
\hline $\mathrm{F}_{3} \ldots \ldots \ldots$ & 0,50 & 265 & 44,6 & 26,78 \\
\hline
\end{tabular}

Quand l'incubation est faite dans de mauvaises conditions, sans retournement des œufs pendant toute la période d'incubation, les différences de la variance entre homozygotes et hétérozygotes augmentent. Les lots d'œufs à forte consanguinité montrent une variabilité particulièrement élevée sous ces conditions de «stress ». Les résultats de cette série d'expériences sont présentés dans le tableau $\mathrm{n}^{\circ} 7$.

Tableau 7. - Taux d'éclosion des œufs après incubation sans retournement

\begin{tabular}{|c|c|c|c|c|}
\hline Génération & $\ll F »$ & $\mathrm{~N}$ & $\begin{array}{l}\text { Pour } 100 \\
\text { éclosion }\end{array}$ & $\begin{array}{l}\text { Coefficient } \\
\text { de variation }\end{array}$ \\
\hline $\mathbf{P} \ldots \ldots \ldots$ & 0 & 1.204 & 44,15 & 14,3 \\
\hline $\mathrm{F}_{1} \ldots \ldots \ldots$ & 0,25 & 1.237 & 27,21 & 24,4 \\
\hline $\mathrm{F}_{2} \ldots \ldots \ldots$ & 0,37 & 1.227 & 16,28 & 56,3 \\
\hline
\end{tabular}

Quand on compare la variance du poids d'embryons de Cailles du sixième et du douzième jour d'incubation, on constate également une variance plus élevée dans les générations consanguines $F_{1}$ et $F_{2}$, comparées avec la souche de référence $P$, non consanguine. Les résultats de ces pesées sont consignés dans le tableau $n^{\circ} 8$. Le poids moyen des embryons à forte consanguinité est plus faible et la variance du poids des embryons consanguins est plus élevée (Boesiger, 1969 b). 
L'homéostase génétique est un phénomène analogue et lié à celui de l'homéostase de développement. Entre les fluctuations et l'hétérogénéité des conditions du milieu et l'hétérogénéité du pool des génotypes d'une population, l'homéostase génétique assure un équilibre qui maintient l'état d'adaptation de la population.

Tableau 8. - Poids d'embryons de Cailles de six et de douze jours

\begin{tabular}{|c|c|c|c|c|c|c|}
\hline \multirow[b]{2}{*}{ Génération } & \multicolumn{3}{|c|}{ Sixième jour } & \multicolumn{3}{|c|}{ Douzième jour } \\
\hline & N & $\begin{array}{l}\text { Moyenne du } \\
\text { poids en mg }\end{array}$ & $\begin{array}{l}\text { Coeffi- } \\
\text { cient de } \\
\text { variation }\end{array}$ & $\mathrm{N}$ & $\begin{array}{l}\text { Moyenne du } \\
\text { poids en mg }\end{array}$ & $\begin{array}{l}\text { Coeffi- } \\
\text { cient de } \\
\text { variation }\end{array}$ \\
\hline P $\ldots$. & 84 & $340,7 \pm 4,4$ & 12,11 & 34 & $3121 \pm 45$ & 8,48 \\
\hline $\mathrm{F}_{1} \ldots \ldots \ldots \ldots$ & 85 & $328,5 \pm 4,8$ & 14,31 & 39 & $3031 \pm 55$ & 11,60 \\
\hline $\mathrm{F}_{2} \ldots \ldots \ldots \ldots$ & 52 & $295,5 \pm 9,8$ & 24,62 & 21 & $2627 \pm 101$ & 26,23 \\
\hline
\end{tabular}

L'homéostase génétique maintient dans chaque population locale les fréquences caractéristiques de différents types structuraux des chromosomes chez les Drosophilides, ou des fréquences particulières de différents facteurs sanguins dans l'espèce humaine, etc...

Quand on exerce une pression de sélection artificielle pour déplacer le niveau de caractères quantitatifs, la sélection naturelle oppose une résistance homéostatique. Quand la pression de sélection artificielle cesse, l'homéostase génétique a tendance à ramener les fréquences de génotypes de la population à l'état antérieur, établi par la sélection naturelle.

Il ne s'agit pas d'approfondir ici les notions de l'homéostase de développement et de l'homéostase génétique, mais simplement d'attirer l'attention sur ces deux facteurs, qui doivent être pris en considération dans la lutte génétique. Il est imporiant que les individus, ainsi que les populations relâchées dans la nature so.ent suffisamment homéostatiques pour pouvoir survivre dans les conditions complexes et dures que subissent les populations naturelles. Il faut insister sur le fait que cela n'est pas le cas, habituellement, pour des animaux élevés au laboratoire.

\section{Génétique et comportement}

Le succès de la lutte génétique dépend dans une assez grande mesure du comportement des individus à relâcher dans les populations nature!les. Les organismes à introduire doivent avoir le même rythme nycthéméral que ceux de la population à infester, les mêmes préférences alimentaires, climatiques, de sites de repos, etc. Il faut surtout 
que les mâles relâchés sachent accomplir une parade nuptiale adéquate. Celle-ci doit être finement ajustée aux habitudes locales des femelles de la population naturelle. La parade nuptiale doit surtout être au moins aussi vigoureuse que celle des mâles de la population naturelle.

Chez D. melanogaster, on trouve de grandes différences de la vigueur des mâles de différentes souches (Boesiger, 1962). Les mâies produits en élevage consanguin sont beaucoup moins vigoureux que ceux qui ont un degré élevé d'hétérozygotie. Leur parade nuptiale est moins persistante et moins intense, leur agressivité sexuelle beaucoup plus faible que celle des mâles hétérozygotes. Les mâles hétérozygotes commencent leur parade nuptiale plus tôt et le délai moyen jusqu'au début de la copulation est plus court que chez les mâles homozygotes.

La vigueur des mâles est déterminée génétiquement. L'influence de certains gènes spécifiques ne peut pas être exclue. Mais la condition génétique la plus importante est le degré d'hétérozygotie. La consanguinité, fréquente dans les élevages de laboratoire, diminue la vigueur des mâles.

Pour la lutte génétique, il est important de savoir que le comportement sexuel que nous constatons au laboratoire, dans des conditions artificielles, est souvent différent de'celui qu'on observe dans la nature.

Au laboratoire, en tube de culture, ou en chambre d'observation, on obtient facilement des croisements interspécifiques, p.e. entre Drosophila melanogaster et Drosophila simulans ou entre Drosophila pseudoobscura et Drosophila persimilis. Dans la nature. ces copulations interspécifiques n'ont jamais lieu! Nous en sommes sûrs, parce qu'on ne trouve pas dans la nature des individus hétérozygotes pour deux types structuraux chromosomiques qui sont spécifiques pour l'une ou l'autre des deux espèces.

Des différences fines de la parade nuptiale, qui échappent à l'observateur humain, peuvent déterminer le succès ou l'échec copulatoire des mâles.

$\mathbf{M}^{\mathrm{me}}$ Ehrman (1966) a montré pour de nombreuses souches de Drosophila paulistorum, qui ne se distinguent pas par des caractères morphologiques, ou par des particularités du comportement sexuel, que l'isolement sexuel est constamment plus grand entre des individus appartenant à deux races différentes, ou espèces naissantes différentes, quand les individus viennent de la même localité. Les individus sympatriques ont donc établi une tendance plus forte à l'iso'ement sexuel, qui se manifeste même dans les conditions artificielles des croisements en chambre d'observation.

Il convient d'insister encore une fois sur le fait, que les espèces naissantes de D. paulistorum qui se croisent au laboratoire, ne le font pas dans la nature. Le comportement sexuel de mâles d'élevage mis en contact au laboratoire avec un certain type de femelles ne permet pas de prévoir leur comportement dans les conditions naturelles et avec d'autres types de femelles.

Ajoutons que la performance d'un certain type de mâles peut dans une certaine mesure être déterminée par leur fréquence relative dans la population (Petit et Ehrman, 1969). 


\section{Conclusion}

La première condition, pour réussir la lutte génétique, est la création expérimentale ou la sélection d'organismes possédant un génotype adéquat. Ce rapport ne traite pas de ce problème fondamental.

Une deuxième condition aussi importante que la première est celle de la compétitivité et de la capacité d'adaptation des organismes à relâcher pour la lutte génétique. Les échecs enregistrés dans la lutte génétique sont certainement en tout premier lieu déterminés par le manque de vigueur et par l'inadaptation écologique des organismes relâchés dans les populations naturelles. Il est indispensable que les animaux relâchés dans la nature puissent s'y maintenir dans de bonnes conditions et qu'ils puissent affronter avec succès la compétition avec les occupants autochtones du biotope, dans lequel la lutte génétique doit se dérouler. Les problèmes de la génétique des populations, brièvement énumérés dans ce rapport, doivent être soigneusement étudiés, pour réussir la lutte génétique. Pour éviter des insuccès décourageants, qui seraient néfastes aux projets d'avenir de la lutte génétique, il est important d'étudier soigneusement la génátique formelle, la génétique des populations sous l'aspect de la génétique écologique (Voir Ford, 1964), et la génétique du comportement, ainsi que la dynamique des populations naturelles. Il est également très important d'étudier les techniques et les bases génétiques de l'élevage des animaux à relâcher, pour obtenir des individus compétitifs et bien adaptés.

On peut résumer les principaux buts de ces travaux en quelques points :

$1^{\circ}$ Les populations naturelles de Moustiques comme celles d'autres organismes, sont hétérogènes. Le pool des génotypes de chaque population locale a une composition particulière, établie par la sélection naturelle en réponse à la gamme spécifique des conditions écologiques multiples et variables de chaque région. Pour que les animaux relâchés dans la population naturelle puissent accomplir la tâche que nous leur assignons, ils doivent être adaptés aux conditions particulières de chaque population naturelle à combattre, ce qui nécessite une étude génétique de ces dernières. Il sera souvent nécessaire, ou au moins utile, de prélever les animaux à manipuler génétiquement dans la population à combattre.

Les différences entre les pools de génotypes des populations locales, ainsi que l'évolution de ces populations au cours du temps, ne se manifestent pas en premier lieu dans la morphologie des organismes, mais dans les systèmes enzymatiques, les types structuraux des chromosomes ou par des particularités du comportement. Les risques d'échec seraient grands, si on ne tenait pas compte de ces facteurs.

$2^{\circ}$ Le pool des génotypes d'une population naturelle est souvent soumis à des fluctuations saisonnières et réversibles et dans certains cas à des évolutions irréversibles au cours des années. Il est indispensable de connaître les changements de la constitution génétique des populations naturelles pour pouvoir adapter continuellement la composition des lots d'animaux à relâcher, puisque nous ne pouvons pas espérer obtenir l'éradication d'une espèce dans un temps court. 
$3^{\circ}$ La vigueur générale des animaux à relâcher, et surtout la vigueur sexuelle des mâles doivent obligatoirement être au moins égales à celles des individus de la population naturelle. Or les animaux élevés au laboratoire sont habituellement beaucoup moins vigoureux que ceux des populations naturelles. Il est donc très important d'étudier les conditions génétiques qui déterminent la vigueur, et de développer des techniques d'élevage et de sélection qui assurent la production d'animaux d'élevage compétitifs.

$4^{\circ}$ Dans les populations naturelles, les organismes doivent habituellement affronter des changements nycthéméraux ou saisonniers des conditions de vie. Dans les populations naturelles l'hétérozygotie des individus et l'hétérogénéité des populations assurent les propriétés homéostatiques indispensables à la survie, sous ces conditions variables. Pour les animaux à relâcher il sera nécessaire de développer des techniques d'élevage sous des conditions très variables et dures, qui provoqueront une forte sélection. Et il sera nécessaire d'éviter la production de lots homogènes d'individus et d'élever au contraire des populations très hétérogènes, qui pourront affronter avec succès les conditions multiples et variables des biotopes naturels.

$5^{\circ}$ Les mâles à relâcher dans la nature doivent trouver les femelles de la population naturelle et réussir à les stimuler autant que le font les mâles autochtones. Ils doivent effectuer une parade nuptiale finement ajustée aux habitudes éthologiques locales. Il n'est nullement assuré que des mâles d'élevage, qui copulent dans les conditions de confinement du laboratoire avec des femelles élevées au laboratoire le feront aussi dans des conditions beaucoup plus complexes d'une population naturelle et avec les femelles de la population naturelle.

$6^{\circ}$ Il est important d'effectuer simultanément avec les recherches de laboratoire mentionnées ci-dessus, et au fur et à mesure de leur développement, des expériences pilotes de lâchers d'animaux sur le terrain pour se rendre compte de leur capacité d'adaptation aux conditions naturelles et de leur efficacité.

$7^{\circ}$ Pour que la lutte génétique soit efficace, il sera sans doute nécessaire d'appliquer plusieurs méthodes génétiques simultanément. Aux méthodes génétiques il faudra joindre d'autres méthodes de lutte biologique, comme par exemple l'introduction de nouveaux prédateurs ou l'introduction d'une espèce proche, non nuisible, qui pourrait entrer en compétition avec le vecteur.

$8^{\circ}$ On ne pourra guère éliminer totalement une espèce de vecteurs hématophages. Les capacités d'adaptation des populations naturelles et l'infestation à partir de régions non traitées nécessiteront un contrôle continuel des populations. Il sera donc utile de développer des techniques simples pour des recensements de routine des populations.

La lutte génétique contre les Arthropodes hématophages est' une tâche difficile et passionnante. Elle permettra de soulager les populations humaines qui souffrent de microorganismes transmis par des vecteurs, sans danger d'intoxication par des substances chimiques. En même temps, elle nous aidera à développer une jeune, mais très importante branche de la biologie, la génétique écologique. 


\section{Bibliographie}

Boesiger (E.), 1962. - Sur le degré d'hétérozygotie des populations naturelles de Drosophila melanogaster et son maintien par la sélection sexuelle. Bull. Biol. de France et de Belgique 96, 3-122.

-, 1963. - Comparaison du nombre de descendants engendrés par des mâles homozygotes et des mâles hétérozygotes de Drosophila melanogaster. C.R. Acad. Sci. (Paris), 257, 531-533.

—, 1965. - Tendances actuelles de la Géné:ique des Populations. In : La Biologie, AubierMontaigne, Paris, p. 147-174.

—, 1968. - Estimation globale de l'âge des femelles de Drosophila melanogaster capturées dans des populations naturelles. C.R. Soc. de Biologie, 162, 358-361.

-, 1969 a. - La structuration génétique des populations de Drosophilides. Bull. Soc. Zool. de France, 94, 535-552.

-, 1969 b. - Homéostase du développement et homéostase génétique. L'Année Biol., 8, 581-614.

-, 1969 c. - Effets de la consanguinité chez la Caille japonaise. Bull. Biol. de France et de Belgique, 103, 285-304.

Dobzhansky (Th.), 1970. - Genetics of the evolutionary Process. Columbia Univ. Press. New York, $505 \mathrm{p}$.

- et Boesiger (E.), 1968. - Essais sur l'Evolution, Masson et $C^{1 e}$, Edit., Paris, 182 p.

- et Epling (C.), 1944. - Contributions to the genetics, taxanomy and ecology of $D$. pseudoobscura and its relatives. Carnegie Inst. Washington Publ., 554.

- et SPASSKY (B.), 1953. - Genetics of natural populations. XXI. Concealed variability in two sympatric species of Drosophila. Genetics, 38, 471-484.

Dubinin (N. P.), Romashov (D. D.), Heptner (M. A.) et Demidova (Z. A.), 1937. - Aberrant polymorphism in Drosophila fasciata Meig. Biol. Zh., Moscou, 6, 311-354.

Ehrman (L.), 1966. - Mating success and genotype frequency in Drosophila. Animal Behaviour, 14, 332-339.

ForD (E. B.), 1964. - Ecological Genetics. Methuen, London.

-, 1965. - Genetic Polymorphism. Faber, London, 101 p.

IVES (P. T.), 1945. - The genetic structure of American populations of D. melanogaster. Genetics, 30, 167-196.

Knipling (E. F.), 1960. - The eradication of the screw-worm fly. Scient. Am., 203, 54-61.

Lerner (M.), 1954. - Genetic Homeostasis. Oliver and Boyd, Edinburgh, 134 p.

Lewontin (R. C.) et HubBy (J. L.), 1966. - A molecular approach to the study of genic heterozygosity in natural populations. II. Amount of variation and degree of he:e rozygosity in natural populations of Drosophila pseudoobscura. Genetics, 54, 595 609.

Pentzos (A.), Boesiger (E.) et Kanellis (A.), 1967. - Fréquences de gènes mutants dans plusieurs populations naturelles de Drosophila subobscura de Grèce. Annales Fac. Sci. Univ. Aristotel. de Thessaloniki, 10, 133-152.

Petit (Cl.) et Ehrman (L.), 1968. - Le rôle de la sélection sexuelle dans l'évolution des populations. L'avantage des types rares dans le groupe willistoni (genre Drosophila, sous-genre Sophophora). Bull. Biol., 102, 433-466. 